\title{
Does the Mind Reflect the Mouth? Sensory Profiling and the Future
}

\section{G. B. DIJKSTERHUIS}

Sensory Science Group, Department of Dairy and Food Science, The Royal Veterinary and Agricultural University, Frederiksberg C, Denmark; Wageningen University and Research Centre, Agrotechnology and Food Innovations, Marketing Science, Department of Economics, University of Groningen, The Netherlands

\section{V. BYRNE}

Sensory Science Group, Department of Dairy and Food Science, The Royal Veterinary and Agricultural University, Frederiksberg C, Denmark

\begin{abstract}
Descriptive Sensory Profiling methods have been used for over half-a-century. As the application of these methods obviously does not occur in a vacuum, we sketch the contextual basis of these methods. A unifying model, illustrating the tasks required from a sensory panellist, is presented with the aim of illuminating some major differences between methods. The history and main developments in the field of descriptive sensory profiling are outlined in a basic pedigree of methods. The authors suggest an approach to sensory profiling where taylor-made, problem oriented, methods are devised and used.
\end{abstract}

Keywords descriptive sensory profiling, future of sensory profiling, sensory panels, sensory psychology, sensory science

\section{INTRODUCTION}

The present article has evolved from a number of discussion sessions between the authors and their colleagues. The work partly was prompted by the sensory vocabulary development work of Byrne, Bak et al. (1999a), Byrne et al. (1999b, 2001a). From this work the authors noted a distinct lack of clarity as to the 'meaning' of various criteria used in choosing sensory terms to describe the sensory characteristics of cooked and chill-stored meat samples that develop an off-flavor referred to as warmedover flavor (WOF, Tims and Watts, 1958). These criteria seem to be connected to the 'profiling philosophy' one adopts for his/her research, which may be a matter of education or even geographical location. For this reason we trace the main ideas found in sensory profiling through the recent history of the field and sketch a basic pedigree (Figure 2). Two main issues appear in all profiling studies, teaching a panel a set of attributes, and subsequently scoring of these, leading us to propose a straightforward scheme of psychological tasks expected of a sensory panellist (Figure 3). Thus, we felt it is timely to reflect on the basis of one of the seemingly most abundant methods of sensory analysis: descriptive sensory profiling. For an overview of

Address correspondence to G. B. Dijksterhuis, Sensory Science Group, Department of Dairy and Food Science, Royal Veterinary and Agricultural University, Rolighedsvej 30, Frederiksberg C, 1958, Denmark. E-mail: gbd@kvl.dk the history of the different types of sensory profiling methods, their main properties, and an extensive literature list we refer to another recent review paper by Murray, Delahunty, and Baxter (2001) about the 'past, present, and future' of descriptive sensory analysis. In our current article we aim to present some critical comments and open up areas of discussion concerning the merits of sensory profiling methodology per se.

What we will call 'descriptive sensory profiling' also goes under other monikers: descriptive (sensory) test, (sensory) profiling, conventional (sensory) profiling, descriptive analysis, and is in fact a name for a class of methods (cf. Murray et al., 2001). We will use the term 'profiling' for reasons of brevity. Moreover, we will use adjectives when we address a specific profiling method, e.g., 'free choice' profiling, or when referring to a specific method, e.g., 'QDA' or 'Spectrum.'

The overall process of performing sensory profiling is broader than the topic of this article. In Figure 1 we systematically summarize the steps involved in sensory profiling studies, including their embedment in the research context. With this we recognize that of course profiling does not take place in a vacuum. Profiling studies are prompted by a certain research question, which can stem from a host of sources. We will not treat all of these, suffice to say that the research question has an obvious impact on the selection of samples and influences both the selection of the panel and the way the panel will be treated prior to profiling proper ('core' in Figure 1). This is indicated in Figure 1 by 


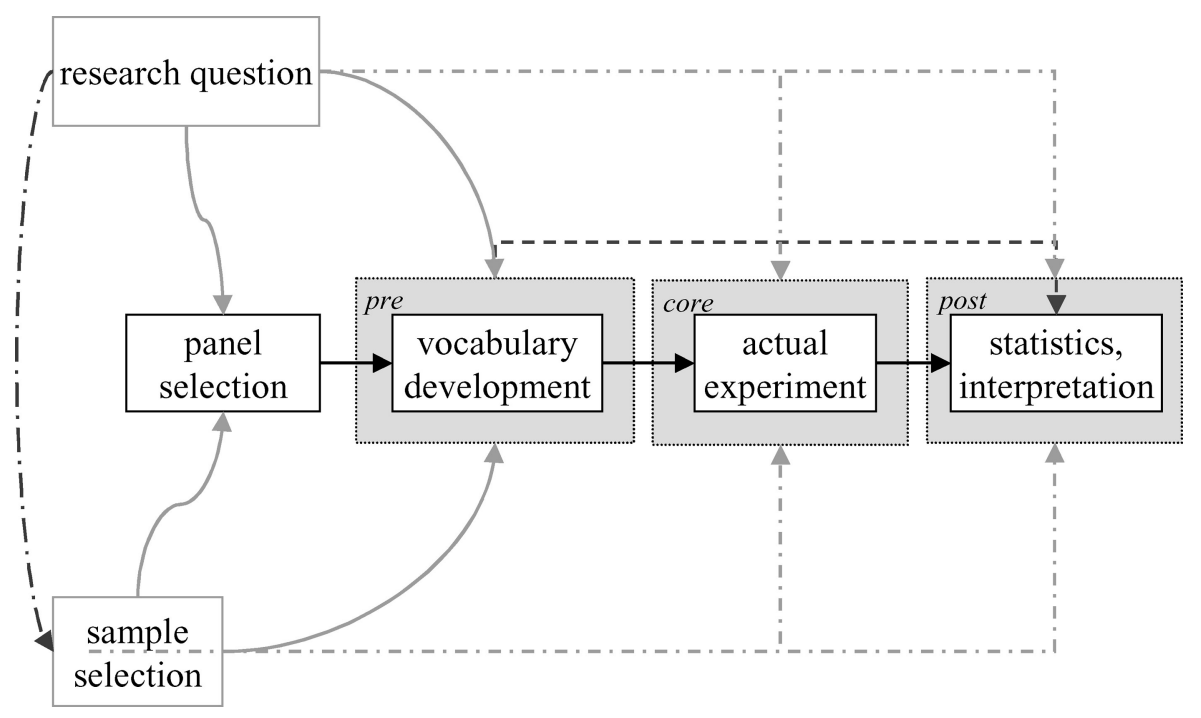

Figure 1 Overview of the steps involved in sensory profiling studies.

arrows emanating from the box 'research question' to the boxes 'panel selection' and 'sample selection.' Another pair of dashed arrows points at the boxes named 'pre,' 'core,' and 'post.' Of course the research question has an effect on the total of the exercise. Even the statistics that are used, their interpretation, and the way the results are presented may depend on the research question and the client. None of the issues highlighted in Figure 1 are specifically our concern in this article (see e.g., Murray et al., 2001 for more details on some of these issues). In ISO (1999, Table 2, p. 4) the steps in establishing a sensory profile are listed, similar to our Figure 1, but with references to relevant ISO documentation.

We will be talking about food, food products, food stimuli, etc., but the field of sensory profiling comprises many studies on non-food items as well. Non-food applications have gained importance as exemplified by publications on a diverse a set of products, such as perfumes, toiletries, fabrics, and more recently automobiles (e.g., Giboreau et al., 2001) and speech through mobile communication channels (Mattila, 2002). Thus, the ideas we describe in this article can be generalized to sensory profiling of non-food items.

\section{Background and Short History of Sensory Profiling}

A number of sources present a short historic overview of profiling methods (Lawless and Heymann, 1998; Powers, 1984; Murray et al., 2001). We have taken the information from several sources and attempt to provide an overview in the form of a pedigree (Figure 2). The pedigree shows that the methods originated in the forties with the creation of the Flavor Profile Method (Cairncross and Sjöström, 1950). Some early variations, like the modified diagram method (Cartwright and Kelly, 1951) and the dilution method (Tilgner, 1962), seem to have come to a dead-end. Note that the time intensity method is a rather early, separate development (see Dijksterhuis and
Piggott, 2001). We do not view it as a 'profiling' method; it is in the pedigree because of its descriptive character, and because it probably helped in spawning later developments. Three major methods subsequently emerged. The texture profiling method (Brandt et al., 1963; Szczesniak, 1963; Szczesniak, et al., 1963), from which the Spectrum method partly originated, combines parts from texture profiling and flavor profiling and covers the whole spectrum of sensory attributes (cf. Rudledge and Hudson, 1990, Meilgaard et al., 1999; Lawless and Heymann, 1998). At the same time Quantitative Descriptive Analysis (QDA) was developed (Stone et al., 1974; Stone and Sidel, 1993), where a quantitative behavioural science (psychophysical) approach was introduced instead of the qualitative consensus approach of flavor profiling. Another method, with a major difference from the previous methods, was Free Choice Profiling (FCP), put forth by Langron (1983), Williams and Langron (1984), and Arnold and Williams (1986). Progressive profiling (Jack et al., 1994) merges the dynamic ideas from time intensity with ideas from flavor and texture profiling, and within the realm of FCP, the selection of attributes was aided by an older psychological method (RGM, Repertory Grid Method, Kelly, 1955).

A number of recent review articles suggested 'Quantitative Sensory Profiling' (QSP, Powers, 1988) or 'Generic Descriptive Analysis' (GDA, Einstein, 1991; Lawless and Heymann, 1998), which essentially is a general heading under which any profiling method could fit, just as the current authors suggest ESP (no pun intended), 'Eclectic Sensory Profiling,' stressing the fact that one should build her/his own tailor made specific problem-oriented profiling method.

\section{Perceptual Matters}

Sensory- and food-scientific research is always prompted by an underlying research question, sometimes based on a practical problem with a food product. If it is recognized that the question 


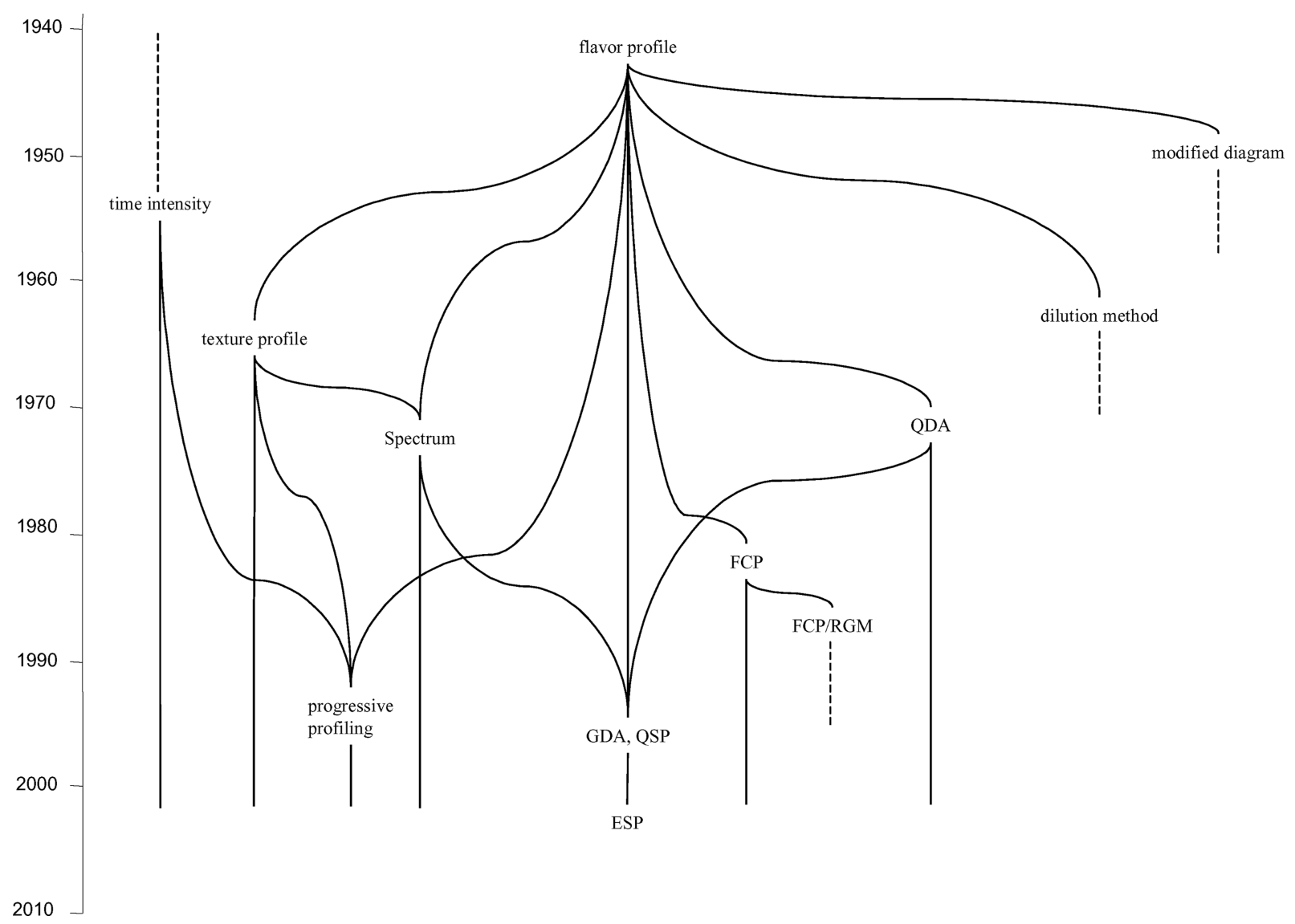

Figure 2 Pedigree schematic of sensory profiling methods.

is of a perceptual nature, which is not always an obvious conclusion, sensory profiling is a way to describe the products. That the acknowledgement of a perceptual cause for a problem with a food product may not be obvious can be illustrated by the case of boar taint. Boar taint is a well known off-flavor in meat from pigs, mostly male pigs, i.e., boars. Substances responsible for this off-flavor have been sought after and successfully identified. First androstenone (Patterson, 1968) was identified in pig meat as clearly producing 'boar-taint.' However, another compound, skatole, was identified soon after (Walstra and Maarse, 1970; Vold, 1970), which was also suggested to be responsible for the off-flavor. There now seem to be two 'camps,' one claiming it's mainly skatole that is responsible for boar-taint, the other favoring androstenone as the major source of boar-taint. Clearly boar-taint poses a perceptual problem. If one compound were solely responsible, the issue would have been resolved. Since it is two compounds, or even more as some suggest (e.g., GarciaRegueiro and Diaz, 1989), the problem is not solely chemical. Perceptual interactions of the two or more compounds may produce the typical off flavor known as boar-taint. Thus, any investigation of boar-taint must include a perceptual component that tries to answer the fundamental question, 'What is boar-taint?' A similar case can be made for WOF (cf. Byrne et al., 1999 a, b, $2001 \mathrm{a}, \mathrm{b}$ ), as referred to in the introduction of this article. One way of investigating the nature of a perceptual problem with a food product, or any other product for that matter, is to employ sensory profiling as an analysis methodology.

\section{Sensory Profiling Studies in the Future}

Considering the fact that descriptive sensory profiling is approximately half-a-century old, we ask ourselves a question about its future. It is relatively easy to perform profiling, provided a sensory panel is available, or else to employ a commercial service which can perform the study. Sometimes a profiling study seems almost like a self-fulfilling prophecy, in that it always gives us data and often the analyzes of the data give results that make 'sense' and are 'interpretable.' However, some of the published results of profiling could have been scrutinized more with respect to the validity and reliability of the findings. This has been said before and has recently been stressed again by Martens and Martens (2001) using various forms of crossvalidation. Replication of several products is an easy way of inferring something about the reliability of the results. It has also been said, in 1962, by Hurley and Cattell that it can be too easy to have a multivariate analysis computer program produce statistics and take it as gospel. Wishful thinking is a risk lurking between the dimensions of many biplots. The need for replicate products or for some form of cross-validation cannot be over-emphasized or under-estimated. 


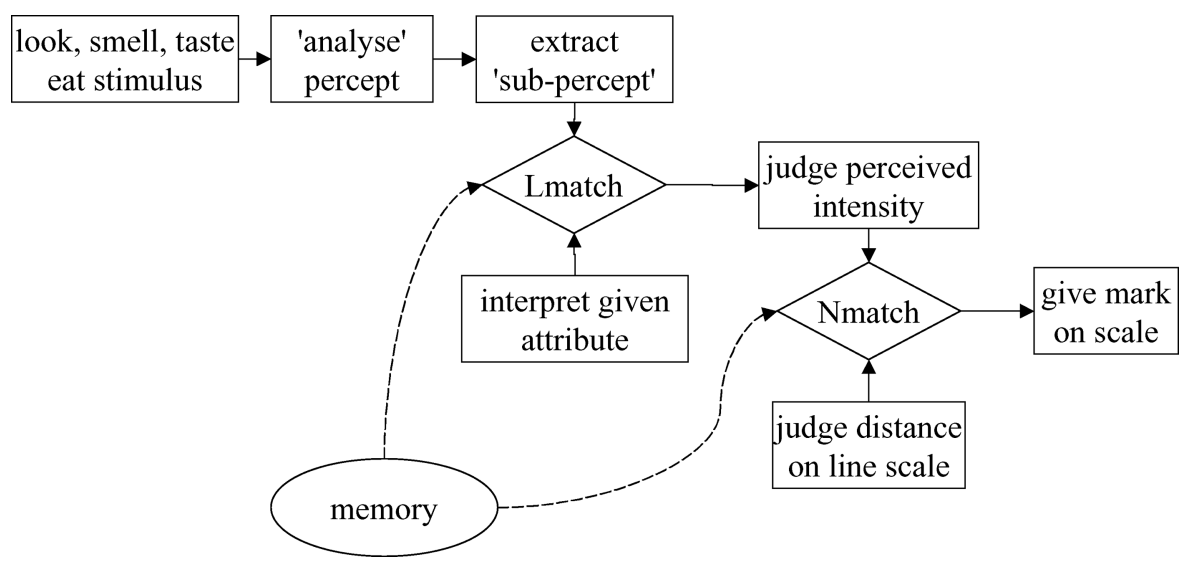

Figure 3 Steps in the process of assessing a food stimulus by a subject in a sensory profiling task ('Lmatch' stands for quaLitative matching; 'Nmatch' for quaNtitative matching).

\section{TASKS INVOLVED IN SENSORY PROFILING}

Below we describe a general phenomenology of a sensory profiling task. The question we address: What exactly is required of a sensory panellist? In Figure 3 we attempt to illustrate several of the tasks a sensory panellist carries out.

A panellist in a sensory profiling study is required to assess a food stimulus visually, through olfaction, by taste, and by eating a food-stimulus and performing a 'perceptual analysis' of the food stimulus. This has to take place with pre-given attributes in mind. The origin of the attributes can be the panel itself, but at the moment of the profiling study proper, they are presented with the attributes for use. The result of this 'perceptual analysis' is a set of specific sub-percepts, one of which must be 'quaLitatively' matched with the attribute in question, or actually with the interpretation a subject has of the attribute ('Lmatch' in Figure 3). The question the subject is trying to answer is whether this specific sub-percept matches the attribute. This requires active memory searches and conscious decisionmaking. The decision depends on the way the subject gauges his/her decisions, which can be lax or strict. Signal Detection Theory (SDT, see Green and Swets, 1966) makes use of this gauging-idea to illustrate the different possible outcomes of a decision making process. The problem in integrating ideas from SDT with sensory profiling methods is that it requires a 'signal' to be present, or not present, in noise polluted surroundings. This is, in principle, not different from sensory profiling, where subjects have to search for a 'signal' from amongst the sensory noise produced by the product under investigation. However the 'signal' in the food product, i.e., an Lmatch is not easily manipulated. It is generally very difficult to construct foods where a certain attribute, the 'signal,' is present, or not present, in order to construct a table with 'hits' and 'misses' of the signal according to SDT. Only in highly artificial model foods would this be feasible. If the subject decides for a positive Lmatch, the quest continues: How strong is the sub-percept in the whole stimulus? The assessor has to revive memories of the intensities of the anchors (if present on the line-scale) and/or interpret the labels at the anchors and judge the distance of the anchors to the position where he/she will finally put his/her mark. This is effectively a cross-modality task, where apparent length of a part of the line-scale is matched to the perceived intensity of a specific attribute. A 'quaNtitative' match is required, hence, 'Nmatch' in Figure 3. Other types of scale (category-scale, magnitude estimation) other than the usually used line-scale (a.k.a. semantic differential or visual analogue scale) essentially trigger the same processes. Figure 3 also illustrates that the subjects memory plays an important role in a profiling task. This warrants research into the role of memory in perception of food and food related stimulation. Memory for food related stimulation (textures, tastes, flavors, odors) is likely to be different from other types of memory. In particular verbalization is shown to be able to deteriorate the content of food-related memory (cf. Melcher and Schooler, 1996); in addition food related memory, at least odour-memory is to a very large extent implicit (cf. Degel, 2000). Mojet and Köster (2001) recently illustrated the incidental manner in which memory for food texture properties seems to work. This can result in distorted memories, e.g., the fattiness of previously encountered foods. The two decision moments, marked Lmatch and Nmatch in Figure 3, are accessible to our intervention as experimenters. This is where we want our training to have effect. The different ways of training (e.g., see Byrne et al., 1999 a, b and 2001) and attempts to affect Lmatch and Nmatch largely define some of the different profiling methods available.

\section{A CONTROVERSY}

Recently a controversy has arose between two different ways of performing descriptive profiling. This discussion appears to have mainly taken place in the U.S.A., which also happens to be the residence of the originators of two 'schools' of sensory profiling methods. One group defends the method developed mainly by Stone et al. (1974, see also Stone and Sidel, 1993); their method has been dubbed 'Quantitative Descriptive Analysis' 
(QDA). The other method, coined 'Spectrum,' was also put forward in the seventies, (see e.g., Meilgaard et al., 1991). The main differences between these two methods lie in what we have called Lmatch and Nmatch in Figure 3. One difference between the two methods lies in the use of a non-technical sensory vocabulary. In the QDA method the vocabulary is based on the terms suggested by the panellists themselves in discussions under supervision of a panel-leader. The other method (Spectrum) prescribes the use of a strict technical sensory vocabulary using reference materials. These matters clearly address Lmatch. The sub-percept should be matched to a term from the sensory vocabulary. The success with which this is achieved may depend on the type of term and the way panellists learned it. The different focus on the vocabulary will result in a different applicability of the profiling results. When the translation to product- and process parameters is secondary to a description of the products in terms of perceptual terms, one would let the panellists use their own words without imposing a strictly technical vocabulary, as in QDA. When it is needed to translate a certain percept to a process parameter or product ingredients, there may result an emphasis on reference material, as in Spectrum. An Lmatch with a clear physical/chemical reference, indeed, may enable this translation.

In our view, a very strict use of a set of references for Lmatch, may limit the validity of the approach for the following reason; interactions of a perceptual nature, which do occur ( $c f$. the above mentioned examples concerning boar taint and WOF), will not easily be mimicked by reference materials. In addition, perceptual interactions are hard to predict. As a result of this, some of the sub-percepts (see Figure 3) may exclusively occur in the food material under study, or even only when it is actually smelled or eaten.

For different reasons, both methods have problems in translating percepts back into product and process parameters, the Spectrum Method by ignoring possible perceptual interactions for which there are no references, and QDA by not providing references for those interactions even if they exist. A rigorous adhering to either method may, thus, result in a sub-optimal result from the profiling exercise. Seen in this way the methods seem partly complementary. This is why we propose to take the strengths of each method to make one's own tailor made profiling method. A similar conclusion was reached by Murray et al. (2001) in their review article on descriptive sensory analysis. In addition, the Spectrum Method prescribes the use of intensity references as well. Insisting on intensity references, aiding an Nmatch fits with the idea of technical control and feedback to processes and ingredients. The Nmatch poses less of a problem in our view, because the perceived intensity of a percept is known to differ enormously between individuals, perhaps even beyond standardizing using references. Statistical standardization, e.g., by level and range correction, may be sufficient to circumvent errors arising from such differences.

There exist more than the above mentioned two profiling methods, but these two seem to take opposing positions in the profiling spectrum, hence, our attention to them. We attempt to summarize the main differences between these and other profiling methods in Table 1. An overview of profiling methods is also provided in Meilgaard et al. (1991), Lawless and Heymann (1998), and by Murray et al. (2001). ISO (1999, Table 1, p. 3) provides a categorization into four main types of sensory profiling methods. We observe that many sensory analysts in the European Union use a generic philosophy for their sensory profiling methodology that we refer to as ESP. They use ideas from several available profiling philosophies and build their own tailor made profiling method ( $c f$. Quantitative Sensory Profiling; Powers, 1988; and Generic Descriptive Analysis; Einstein, 1991; Lawless and Heymann, 1998).

\section{A Note on Statistics}

In profiling an ANOVA on the individual attributes averaged over the panellists is a standard way of assessing differences between the products. We have witnessed a development, or at least a change, of the statistical tools for profiling data from the univariate to the multivariate, and simultaneously, a parallel movement from the confirmatory to the exploratory. The classic statistical pre-conception has often been that one has to be able to generalize to a population, predict future outcomes, or both. If this is the aim, one, indeed, may need inferential statistics including assumptions about, distributions, homoscedasticity, etc.. However, in sensory profiling the aim is often not prediction or generalization, it is description of a specific set of samples. In such cases the apparatus of statistical inference may not be needed in its strict sense, and any exploratory use of statistical methods, e.g., the gamut of exploratory multivariate methods of data analysis, is justified (cf. Dijksterhuis and Heiser, 1995).

\section{DISCUSSION}

The name is self-explanatory, 'descriptive' profiling, meaning that things are described. There is nothing inherently inferior in descriptions; Newtonian mechanics is based on descriptions only ('I make no hypotheses.'-SIR Isaac Newton), but in the case of perception science, we seem to be living in an era where explanations are sought after vigorously, partly as a result of progress in techniques for studying brain-processes. Improvements of profiling methodology have been made in the statistics used for the analysis and in the methodologies of training, but not in the substance of the profiling methods themselves. We (the sensory/consumer science community) have seen variant methods of profiling. Since Free Choice Profiling, has been introduced (Arnold and Williams, 1986), we have taken more interest in the dynamic aspects of food perception in Time Intensity methods (cf. Dijksterhuis and Piggott, 2001) and combined those into progressive profiling (e.g., Jack et al., 1994), but neither of these bring new light to explanations versus descriptions. It is our position that it's timely to attempt to move from the mere descriptive to the explanatory, i.e., employ studies to find 


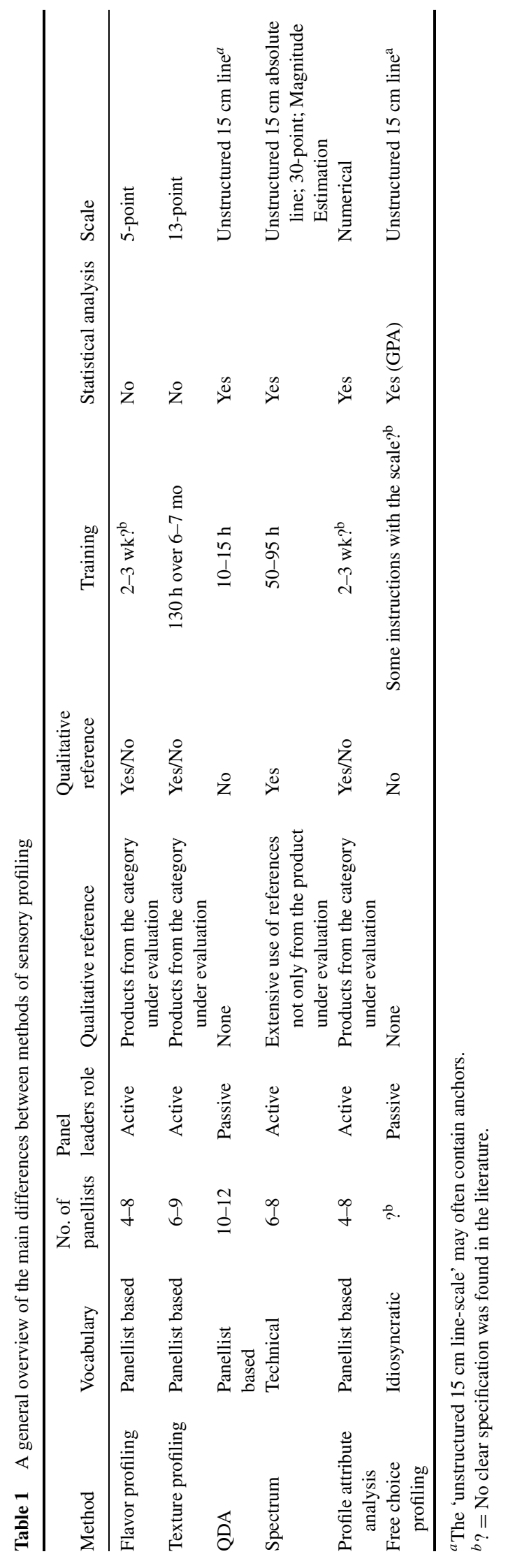


out what the reason can be that a certain attribute is perceived with a certain intensity and another may not; since the subject matter of profiling is about perception, this means a rigorous linking of, parts of, sensory science with perception science.

As we concluded from our schematic in Figure 3, the two decision processes (Lmatch and Nmatch) involved in profiling require conscious action by the panellists; they are required to enter an analytical mode of thinking. This is fine with descriptive methods as they are, but we realize that most of our perceiving food related stimulation is probably not conscious and hence, is not addressed directly by profiling methodology (cf. Wolf Frandsen, et al., 2002; Köster, 2003). Profiling studies are, indeed, not tuned to study preferences or food choice-behavior; most sensory text-books even forbid it. Sensory profiling studies cannot be interpreted so that they teach us about consumer behavior, per se. It is an analytical measurement instrument and, because it uses humans, a valid one with respect to the perception of products' properties, but not with respect to any food-related behavior of consumers. The latter is addressed by affective tests, or perhaps even more validly, by experiments in which actual observed behavioral acts constitute the data collected.

\section{CONCLUSION}

Descriptive Sensory Profiling is a very powerful method and is applied to an ever increasing extent in many surroundings, both scientific and applied. Some of the discussions regarding the exact way sensory profiling is performed fail, in our view, to address the core of the method. The core being that it is presupposed that a 'trained' set of assessors, become a reliable, and valid, measuring instrument. This supposition of course depends on the quality of the sensory training carried out prior to profiling. Moreover, generalization of profiling results to a consumer population is very difficult, because sensory profiling is limited in that it focuses on non-affective parts of food perception. Actual food choice behavior is affective-('liking') based, and the ultimate challenge often is to predict food choice. In a scientific era where the focus seems to shift from descriptions to explanations, one has to be ready for new methods and realize the limitations of current methods. Thus, it must be said that Profiling methods are still under development. The history of the methods show some clear lines of descent, and a recent, eclectic, focus on generic profiling methods. We do not intend to gloss over the merits of sensory profiling that we subscribe to, but we would like to challenge our colleagues by drawing attention to the need for validation, improvement, and experimental creativity in future applications of sensory profiling.

\section{ACKNOWLEDGEMENTS}

The work of author Dijksterhuis is part of the Føtek Program supported by the Danish Dairy Research Foundation and the Danish Government. Author Byrne acknowledges the 'ConSense' and 'Sense-Index' projects in relation to the present ar- ticle and the financial support of the The Directorate for Food, Fisheries and Agri Business (DFFE) under the Danish Ministry of Food, Agriculture, and Fisheries. We thank colleagues for fruitful and critical discussions of our ideas and of this article. Especially we thank Karina Rossen for the work she did for a literature study as part of her M.Sc. Education. An anonymous reviewer is thanked for constructive comments on the article.

\section{REFERENCES}

Arnold, G.M., and Williams, A.A. 1986. The use of Generalised Proceruustes techniques in sensory analysis. In: Statistical procedures in food research. J.R. Piggott, (Ed.) pp. 233-253. Elsevier Applied Science: London.

Brandt, M.A., Skinner, E., and Coleman, J. 1963. Texture profile method. Journal of Food Science, 28:404-410.

Byrne, D.V., Bak, L.S., Bredie, W.L.P., Bertelsen, G., and Martens, M. 1999a. Development of a sensory vocabulary for warmed-over flavour: Part I. In porcine meat. Journal of Sensory Studies, 14:47-65.

Byrne, D.V., Bredie, W.L.P., and Martens, M. 1999b. Development of a sensory vocabulary for warmed-over flavor. II. In chicken meat. Journal of Sensory Studies, 14:67-78.

Byrne, D.V., O'Sullivan, M.G., Dijksterhuis, G.B., Bredie, W.L.P., and Martens, M. 2001a. Sensory panel consistency during development of a vocabulary for warmed-over flavour. Food Quality and Preference, 12:171-187.

Byrne, D.V., Bredie, W.L.P., Bak, L.S., Bertelsen, G., Martens, H., and Martens, M. 2001b. Sensory and chemical analysis of cooked porcine meat patties in relation to warmed-over flavour and pre-slaughter stress. Meat Science, 59:229-249.

Byrne, D.V., Bredie, W.L.P., Mottram, D.S., and Martens, M. 2002. Sensory and chemical investigations on the effect of oven cooking on warmed-over flavour development in chicken meat. Meat Science, 61:127-139.

Byrne, D.V., O'Sullivan, M., Bredie, W.L.P., and Martens, M. 2003. Descriptive sensory profiling and physical/chemical analyses of warmed-over flavour in meat patties from carriers and non-carriers of the $\mathrm{RN}^{-}$allele. Meat Science, 63:211-224.

Cairncross, S.E., and Sjöström, L.B. 1950. Flavour profiles-A new approach to flavour problems. Food Technology, 4:308-311.

Cartwright, L.C., and Kelly, P. H. 1951. Sharper flavor ratings with improved profile test. Food Engineering, 23:71-73, 215.

Caul, J.F. 1957. The profile method of flavour analysis. Advances in Food Research, 7:5-37.

Civille, G.V., and Liska, I.H. 1975. Modifications and applications to foods of the General Foods sensory texture profile technique. Journal of Texture Studies, 6:19-31.

Degel, J. 2001. Implicit memory for odours. Ph.D. Thesis. University of Utrecht, The Netherlands.

Dijksterhuis, G.B., and Heiser, W.J. 1995. The role of permutation tests in exploratory multivariate data analysis. Food Quality and Preference, 6:263-270.

Dijksterhuis, G.B., and Piggott, J.R. 2001. Dynamic sensory measurements. Trends in Food Science and Technology, 11:284-290.

Einstein, M. 1991. Descriptive techniques and their hybridisation. In. Sensory science theory and applications in foods. H.T. Lawless and B.P. Klein (pp. 317-338). Dekker: New York.

Garcia-Regueiro, J.A., and Diaz, I. 1989. Evaluation of the contribution of skatole, indole, androstenon and androstenols to boar-taint in back fat of pigs by HPLC and capillary gas chromatography (CGC) Meat Science, 25:307316.

Giboreau, A., Navarro, S., Faye, P., and Dumortier, J. 2001. Sensory evaluation of automotive fabrics: The contribution of categorization tasks and non verbal information to set-up a descriptive method of tactile properties. Food quality and preference, 12:311-322.

Green, D.M., and Swets, J.A. 1966. Signal Detection Theory and Psychophysics. Wiley: New York. 
Hurley, J.R., and Cattell, R.B. 1962. The Procrustes Program: Producing direct rotation to test a hypothesized factor structure. Behavioural. Science, 7:258262.

ISO. 1985. International Standard 6564.Sensory analysis-MethodologyFlavour profile methods. Ref. No. ISO 6564:1985 (E). International Organization for Standardization. Genève.

ISO, 1994. International Standard 11035.Sensory analysis-Identification and selection of descriptors for establishing a sensory profile by a multidimensional approach. Ref. No. ISO 11035:1994 (E). International Organization for Standardization. Genève.

Jack, F.R., Piggott, J.R., and Paterson, A. 1994. Analysis of textural changes in hard cheese during mastication by progressive profiling. Journal of Food Science, 59:539-543.

Kelly, G.A. 1955. The psychology of personal constructs. 2 Vols. Basic Books: New York.

Köster, E.P. 2003, The psychology of food choice: Some often encountered fallacies. Food Quality and Preference, 14:359-373.

Langron, S.P. 1983. The application of Procrustes statistics to sensory profiling. In: Sensory quality in food and beverage: Definition, measurement and control. A.A. Williams and R.K. Atkin (Eds.). (pp. 89-95). Ellis Horwood Ltd: Chichester.

Lawless, H.T., and Heymann, H. 1998. Sensory evaluation of foods: principles and practices. Chapman and Hall: New York.

Martens, H., and Martens, M. 2001. Validation X? Y??. In: Multivariate Analysis of Quality. An Introduction. pp. 177-205 J. Wiley and Sons Ltd: London.

Mattila, V.-V. 2002. Semantic analysis of speech quality in mobile communication: Descriptive language development and mapping to acceptability. (Abstract) S10-1. 6th Sensometrics Conference, July 31-August 2, 2002. Dortmund, Germany.

Meilgaard, M., Civille, G.V., and Carr, B.T. 1999. Sensory Evaluation Techniques. 2nd edition. CRC Press, Inc: Boca Raton, Florida.

Melcher, J.M., and Schooler, J.W. 1996. The misremembrance of wines past: Verbal and perceptual expertise differentially mediate verbal overshadowing of taste memory. Journal Of Memory And Language, 35:231245 .

Mojet, J., and Köster, E.P. 2002. Texture and flavour memory in foods: An incidental learning experiment. Appetite 38:110-117.
Murray, J.M., Delahunty, C.M., and Baxter. 2001. Descriptive sensory analysis: Past, present, and future. Food Research International, 34:461-471.

Patterson, R.L.S. 1968. 5 $\alpha$-antrost-16-ene-3-one, compound responsible for taint in boar fat. Journal of the Science of Food and Agriculture, 19:31-37.

Powers, J.J. 1988. Current practices and applications of descriptive methods. In: Piggott, J. Sensory analysis of foods. Elsevier Applied Science Publishers: London.

Rutledge, K.P., and Hudson, J.M. 1990. Sensory evaluation: Method for establishing and training a descriptive flavor analysis panel. Food Technology, 44:78-84.

Stone, S., Sidel, J.L., Oliver, S., Woolsey, A., and Singleton, R.C. 1974. Sensory evaluation by Quantitative Descriptive Analysis. Food Technology, 28:24-34.

Stone, H., and Sidel J.L. 1993. Sensory Evaluation Practices. San Diego, Academic Press Inc: California.

Szczesniak, A.S. 1963. Classification of textural characteristics. Journal of Food Science, 28:385-389.

Szczesniak, A.S., Brandt, M.A., and Friedman, H.H. 1963. Development of standard rating scales for mechanical parameters of texture and correlation between the objective and the sensory methods of texture evaluation. Journal of Food Science, 28:397-403.

Tilgner, D.J. 1962. Dilution tests for odor and flavor analysis. Food Technology, 16:26-29.

Tims, M.J., and Watts, B.M. 1958. Protection of cooked meats with phosphates. Food Technology, 12:240-243.

Vold, E. 1970. Fleischproduktionseigenschaften bu Ebern und Kastraten. IV. Olganoleptische und gaschromatografische Untersuchungen Wasserdampflfiüchtiger Stoffe des Rückenspeckes von Ebern. Meldinger Nordlandbruckhoegskole, 49:1-25.

Walstra, P., and Maarse, H. 1970. Onderzoek geslachtsgeur van mannelijke mestvarkens (Study of sex-odour of male pigs. in Dutch). IVO-report C-147, pp 30.

Williams, A.A., and Langron, S.P. 1984. The use of free-choice profiling for the evaluation of commercial ports. Journal of the Science of Food and Agriculture, 35:558-568.

Wolf Frandsen, L., Dijksterhuis, G.B., Brockhoff, P.B., and Martens, M. 2003. Subtle differences in milk: Comparison of an analytical and an affective test, Food Quality and Preference, 14:5-6, 515-526. 